\title{
Architecting Security with Paradigm
}

\author{
S. Andova ${ }^{1}$, L.P.J. Groenewegen ${ }^{2}$, J.H.S. Verschuren ${ }^{2}$, and E.P. de $\operatorname{Vink}^{1}$ \\ 1 Department of Mathematics and Computer Science \\ Technische Universiteit Eindhoven, The Netherlands \\ ${ }^{2}$ LIACS, Leiden University, The Netherlands
}

\begin{abstract}
For large security systems a clear separation of concerns is achieved through architecting. Particularly the dynamic consistency between the architectural components should be addressed, in addition to individual component behaviour. In this paper, relevant dynamic consistency is specified through Paradigm, a coordination modeling language based on dynamic constraints. As it is argued, this fits well with security issues. A smaller example introduces the architectural approach towards implementing security policies. A larger casestudy illustrates the use of Paradigm in analyzing the FOO voting scheme. In addition, translating the Paradigm models into process algebra brings model checking within reach. Security properties of the examples discussed, are formally verified with the model checker mCRL2.
\end{abstract}

\section{Introduction}

Characteristic for software security problems is, all details matter [2]. Such details fall into several categories centered round the software that must be secure. First: computational details, purely internal to a single component of the software. Second: interaction details between the various components of the software. Third: interaction details between the software and relevant other application software. Fourth: interaction details between the software and the lower level inner world of machine software and hardware. Fifth: interaction details between the software and the outer world of its human stakeholders. Finally, in a recurrent manner: sets of these categories can be found again, centered round relevant other pieces of software, hardware or stakeholders.

In general software development, it has become standard to integrate such diverse categories of computation and interaction coherently within one model, at least at a global level. To that aim, architecture description languages and architectural frameworks are used, cf. [16, 24,28], comprising not only the software application level and technical infrastructure level, but also the organization level constituting the habitat of the software. An architectural model in such a language succeeds in giving a clear and coherent overview of the problem situation at hand, but its interaction details remain declarative mainly, as e.g. serviceoriented architectures do not usually go into the details of an orchestration or choreography of a service.

In the context of security, where all details matter, an architectural approach might be seriously hampering insight into the quality of the solution. By its 
global nature, an architectural description does not readily express every detail of the security problem situation. And even worse, architectures are weak in clarifying operationally how interaction occurs and what behavioural consequences may arise. This is not amazing as even in detailed UML models for software design, dynamic consistency is a problem far from being solved within the UML language [27]. However, interaction categories dominate the listing given above. So, behavioural interaction details are of the greatest importance.

Very often, coordination languages are successfully used for interaction issues, also in relation to architectures. In the context of security it is argued however, coordination solutions are not so easy to apply. Where security is generally oriented towards restriction of dynamics by effectively prohibiting and preventing unwelcome behaviour of participants and intruders, coordination is rather more oriented towards broadening dynamics by efficiently establishing a larger behavioural scope through collaboration, see [44].

For security problems of a larger size, the above disadvantages of architectures or coordination languages, are even more prominent: more details that matter, more coordination directed to even more restrictive dynamics. Nevertheless, we propose the coordination modeling language Paradigm (see e.g. [19, 18, 4]) both for architecting and for coordinating solutions to larger security problems. Paradigm's architecting is done by splitting the problem situation into well-chosen collaborations, characterized each through a separate protocol. Protocol dynamics, although global, are kept dynamically consistent with detailed dynamics of collaborating participants, through well-defined roles. Paradigm's specification of coordination solutions is in terms of consistency rules forming protocols, typically formulated as constraint orchestration or as constraint choreography. Thus, additional effective restriction-on-purpose of collaboration protocol dynamics towards a solution for security issues, fits well with Paradigm's usual orchestration or choreography of constraints.

To underpin the above claims, the sequel is organized as follows. In Section 2 we introduce Paradigm by means of a small secure email example, with a light architectural flavor already present. Section 3 presents Paradigm's constraint architecting by using a larger e-voting example, the FOO voting scheme. Here it is not only specified how one individual voter is to be handled, but also how potential voters are to be hoarded as an ensemble. Section 4 addresses formal verification of the Paradigm models, through model checking on the basis of a process algebraic translation. Related work is discussed in Section 5. Section 6 gives conclusions.

\section{An email example: Paradigm explained}

In this section we briefly explain the key notions of the coordination modeling language Paradigm. A simple case dealing with security policies for encryption of email messages is used as a running example. The exposition should provide sufficient understanding of Paradigm for the subsequent sections, in particular for Section 3, where we discuss a more extensive voting scheme. For more detailed introductions to Paradigm we refer to $[17,19,43]$. 
Let us consider the following situation. In the R\&D laboratory of a company, confidential research is taking place. A document security policy applies to email communication. It states that email addressed to colleague researchers may be signed and encrypted, dependent on the security label of the email content or attachments. Additionally, mail directed to recipients outside the lab are mandatorily encrypted. To support the cryptographic algorithms used, a public key infrastructure has been set up.

Some workers use a PDA for email communication. For this, a locationdependent security policy is in place, demanding all email traffic to be encrypted, when sent using the PDA outside of the premises of the laboratory. Antennas at the exits of the lab send a signal to the PDA, caught by the security module on the PDA, upon entering or leaving the grounds. The email client of the PDA will automatically encrypt all messages when being outside, and provides optional encryption when being inside. However, encryption in particular, substantially consumes battery power. Therefore, as an exception to the rule, for email of a low security label, the PDA owner may override the obligation to encrypt when being outside. Upon completion of sending an email, the PDA switches back to the default mode of encryption, optional or mandatory encryption when inside, mandatory when outside, whichever applies.

In view of modeling coordination solutions in terms of behavior influencing, Paradigm has five key notions: STD, phase, (connecting) trap, role and consistency rule. We shall introduce them guided by the R\&D lab example.

Every Paradigm model is built from STDs, purely sequential behavioral units.

- A state-transition diagram $(S T D)$ is a triple $\langle\mathrm{ST}, \mathrm{AC}, \mathrm{TS}\rangle$ with $\mathrm{ST}$ the set of states, $\mathrm{AC}$ the set of actions and $\mathrm{TS} \subseteq \mathrm{ST} \times \mathrm{AC} \times \mathrm{ST}$ the set of transitions or steps. A step $\left(x, a, x^{\prime}\right) \in \mathrm{TS}$ is said to be a step from $x$ to $x^{\prime}$, notation $x \stackrel{a}{\rightarrow} x^{\prime}$.

Thus, an STD is just a labeled transition system (LTS), rather like a very simple, purely sequential state machine in UML.

(a)

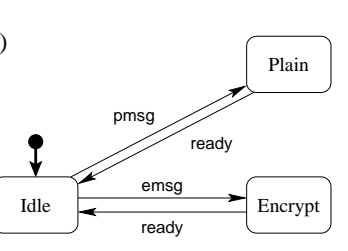

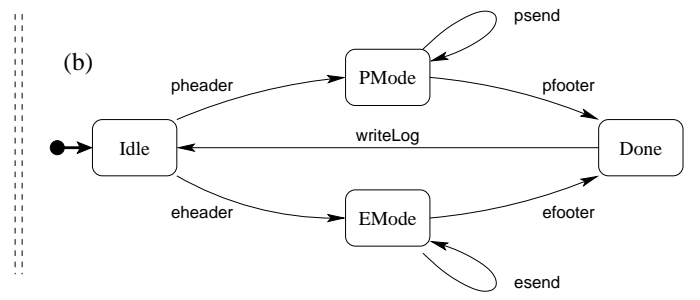

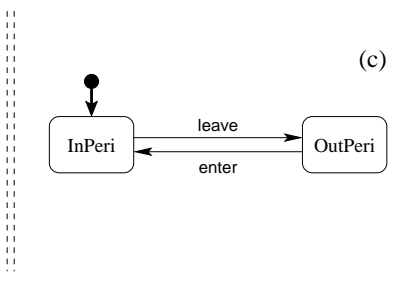

(c)

Fig. 1. Basic STDs: (a) PDA, (b) email client EMC, (c) security module SM.

Modeling the above R\&D lab encryption aspects with Paradigm entails, three components are being distinguished. Their dynamics are modeled through one STD each: a PDA, an email client on the PDA, a security module on the PDA. See Figure 1abc visualizing the three respective STDs in UML-style: rounded rectangles as states and a black-dot-and-arrow pointing to a starting state. The 
user of the PDA can send a message either in plain mode by moving to state Plain or in encryption mode by moving to state Encrypt. After the message has been sent, PDA returns to the starting state Idle. (Below we will refine this.) The email client EMC, when asked to send a message, splits the message into blocks and transmits them with additional header and footer. It does so, either in plain mode, state PMode, or in encryption mode, state EMode. After arrival in state Done, the email client returns to its state Idle. The security module SM shuttles between the two states InPeri and OutPeri, registering whether the PDA is inside or outside the security perimeter.

The main coordination modeling issue is, to organize the mutual influencing of the components such that the security policies are respected. For example, while PDA resides in state Encrypt, the email client EMC should remain restricted to taking steps where sending of the header, of the separate blocks and of the footer occurs in encrypted mode only. To that aim, Paradigm provides the notions of a phase of an STD and of a trap of a phase, both notions serving as temporary constraint on the STD's dynamics, i.e. on the choice there is for taking steps.

- A phase of STD $\langle\mathrm{ST}, \mathrm{AC}, \mathrm{TS}\rangle$ is an $\mathrm{STD} S=\langle$ st, ac, ts $\rangle$ such that st $\subseteq \mathrm{ST}$, ac $\subseteq \mathrm{AC}$ and $\mathrm{ts} \subseteq\left\{\left(x, a, x^{\prime}\right) \in \mathrm{TS} \mid x, x^{\prime} \in \mathrm{st}, a \in \mathrm{ac}\right\}$.

A phase of an STD is itself an STD, actually a subSTD of the STD it is a phase of. As such, a phase of an STD is meant to express a temporarily valid dynamic constraint imposed on the STD it is a phase of. Visualized, a phase is an STDlike fragment of the original, larger STD preserving the form of the original in the fragment. See Figure 2abc. Apart from the extra rectangles to be discussed below, each figure part represents one phase of the full EMC in Figure 1b, viz. PSend, ESend and Finished. PSend gives the behavior needed for plain sending; ESend singles out the behavior needed for encrypted sending; Finished represent the behavior needed for getting prepared for whatever next sending after having closed the last sending properly.

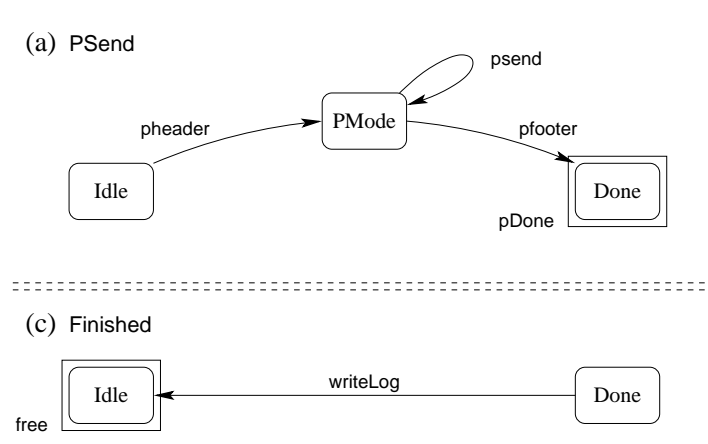

(b) ESend

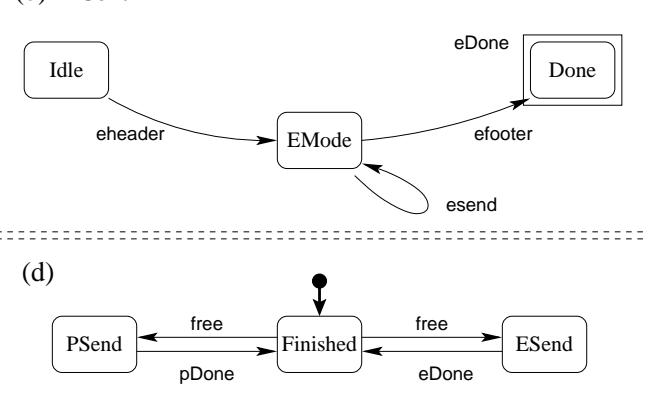

Fig. 2. (a-c) phases PSend, ESend and Finished, (d) role EMC(DoPo). 
Traps, the other dynamic constraint notion of Paradigm, are stepping stones for switching from one phase to another.

- A trap $t$ of phase $S=\langle$ st,ac, ts $\rangle$ is a non-empty set of states $t \subseteq$ st such that $x \in t$ and $x \stackrel{a}{\rightarrow} x^{\prime} \in$ ts imply $x^{\prime} \in t$. The trap $t$ of $S$ connects phase $S$ to another phase $S^{\prime}=\left\langle\mathbf{s t}^{\prime}, \mathrm{ac}^{\prime}, \mathrm{ts}^{\prime}\right\rangle$, notation $S \stackrel{t}{\rightarrow} S^{\prime}$, if $t \subseteq \mathbf{s t}^{\prime}$. This is called a phase transfer. If $t=\mathrm{st}, t$ is called the trivial trap of $S$.

A trap of a phase is a subset of the states of the phase, such that once entered, the subset cannot be left as long as the phase remains imposed. A trap represents a second type of dynamic constraints, committed to by a phase, through its own dynamics: within a phase, the entering of a trap is irrevocable, thus marking the beginning of a final stage of the phase. A trap often serves as a guard for a phase change of the basic STD, i.e. as a guard for changing the constraint currently imposed into a constraint imposed next. In such a case, the trap has to be connecting to the phase aimed at next.

A trap is visualized as a polygon surrounding the states belonging to the trap. More concretely, the three small rectangles in each of the diagrams in Figure 2a-c represent a trap of the particular phase, named pDone, eDone and free, respectively. Below we shall see examples of larger (connecting) traps. Normally, as is the case here, trivial traps are not drawn, unless serving as connecting trap. Note, trap pDone is connecting from PSend to Finished, trap eDone is connecting from ESend to again Finished and trap free is connecting from Finished to PSend as well as to ESend. Hence, phase transfers PSend $\stackrel{\text { pDone }}{\longrightarrow}$ Finished, ESend $\stackrel{\text { eDone }}{\longrightarrow}$ Finished, Finished $\stackrel{\text { free }}{\longrightarrow}$ PSend and Finished $\stackrel{\text { free }}{\longrightarrow}$ ESend are well-defined.

From phase transfers a concrete role STD can be constructed. Roles are generally defined in terms of phases and of traps thereof, belonging each to a well-chosen set, referred to as a partition.

- A partition $\pi=\left\{\left(S_{i}, T_{i}\right) \mid i \in I\right\}$ of an STD $Z$ is a set of phases $S_{i}$ of $Z$ and a set $T_{i}$ of traps of $S_{i}$. The role or global STD at the level of partition $\pi$ is an STD $Z(\pi)=\langle\mathrm{GST}, \mathrm{GAC}, \mathrm{GTS}\rangle$ with GST $\subseteq\left\{S_{i} \mid i \in I\right\}, \mathrm{GAC} \subseteq \bigcup_{i \in I} T_{i}$ and GTS $\subseteq\left\{S_{i} \stackrel{\mathrm{t}}{\rightarrow} S_{j} \mid i, j \in I, t \in \mathrm{GAC}\right\}$ a set of phase transfers. $Z$ is called the detailed STD underlying global STD $Z(\pi)$, the $\pi$-role of $Z$.

Thus, a role of an STD is based on a partition, a particular set of phases of the STD and of connecting traps between them. Here, a connecting trap marks the readiness of a phase to be changed into another phase within the role. The role is to provide a consistent and global view on the ongoing detailed dynamics of the underlying STD. If phases and traps have been chosen well, such a global view expresses precisely the dynamics essential for coordinating the underlying STD via its role. On the one hand, the current state of the role STD, being a phase, imposes the constraint being relevant for the coordination at that moment, on the underlying detailed dynamics. On the other hand, the current detailed state belonging to a trap, is a commit towards the ongoing coordination: the detailed STD shall stay within the trap until a next phase is imposed. In this manner, a role remains dynamically consistent with the underlying detailed STD. 
More concretely, Figure 2d presents the role EMC(DoPo) for implementing the coordination consequences of the document security policy for the email client EMC. The role in Figure 2d has the three phases PSend, ESend and Finished as its states and it has the three connecting traps pDone, eDone and free as its actions. Thus, partition DoPo consists of the phases and traps from Figure 2a-c, together with the three trivial traps (not drawn).

The key idea is, at each point in time a component not only is in one of the states of its detailed STD, but for every role the component has, at each point in time the component is also in one of the phases of that role. Therefore, to maintain consistency, in Paradigm a detailed transition can only be made, if allowed by every current phase of its roles, compliant with the current constraints imposed. In addition, in Paradigm a global transition can only be made, if the component's current detailed state belongs to the trap labeling the global transition, currently entered and hence committed to. For example, if the email client is in detailed state Idle as well as in global state ESend, the detailed STD cannot take the transition from Idle to PMode. Hence, from Idle, sooner or later it is to take the step to EMode, if any, and possibly much later the step from EMode to Done. Only then a connecting trap is entered, viz. trap eDone connecting from ESend to Finished, whereupon at the role level sooner or later the global transition labeled eDone is to be taken from phase ESend to phase Finished.

The control of actually taking a role step, is governed by the consistency rules. Via a consistency rule other roles are taken into account, relating the behavior of individual components depending on the coordination one wants to achieve. A consistency rule synchronizes single steps of detailed and global STDs as follows: per consistency rule at most one detailed step and arbitrarily many global steps from different roles. As general consistency rule format we use:

detailed transition $*$ global transition, ..., global transition

Relating to a consistency rule format we use the following terminology (cf. [43]).

- protocol step: consistency rule with at least one global transition

- orchestration step: protocol step with a detailed transition

- choreography step: protocol step without a detailed transition

- protocol: a set of protocol steps

- choreography: a protocol with choreography steps only

- orchestration: a protocol with at least one orchestration step

- protocol conductor: detailed STD with a transition occurring in the protocol

- protocol participant: detailed STD having a role in the protocol. 
We have four consistency rules, together called the PlainOrEncrypt protocol, that define the DoPo role of the email client EMC. (Below we shall refine it).

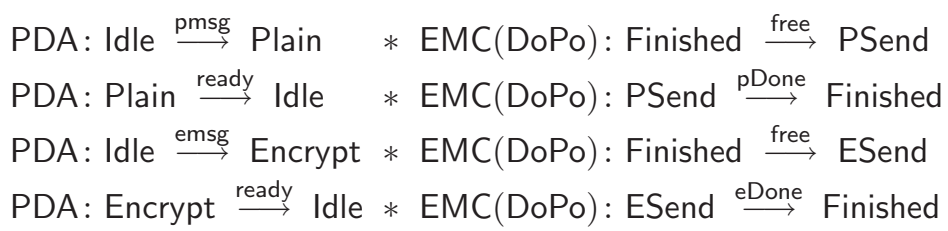

This protocol certainly is an orchestration. PDA is present as conductor in every consistency rule of it. The first rule, for example, is operationally interpreted as follows: PDA, when in state Idle and if allowed to do so by every role of it, can make a pmsg transition to state Plain, if also EMC, residing in phase Finished of role DoPo, has reached trap free and thus can make a transfer to phase PSend. This way, the detailed step of conductor PDA is coupled to the phase transfer or global step of participant EMC in role DoPo. The four consistency rules specify: PDA is conducting EMC in sending either in plain or encrypted as well as in preparing for sending again; EMC is notifying PDA when such conducting has led to the result aimed at.

We want the security module to conduct PDA. The alternative of the security module conducting the email client directly is possible too, but not done here. Thus, we have the following two consistency rules, collectively referred to as the InOrOut protocol. (A refined version of the protocol is given below.)

$$
\begin{aligned}
& \text { SM : InPeri } \stackrel{\text { leave }}{\longrightarrow} \text { OutPeri } * \text { PDA }(\text { LoPo }): \text { EncryptSome } \stackrel{\text { triv }}{\longrightarrow} \text { EncryptAll } \\
& \text { SM : OutPeri } \stackrel{\text { enter }}{\longrightarrow} \text { InPeri } * \text { PDA }(\text { LoPo }): \text { EncryptAll } \stackrel{\text { triv }}{\longrightarrow} \text { EncryptSome }
\end{aligned}
$$

The PDA is supposed to have a role LoPo, to deal with the location security policy. Within this role there are two phases, EncryptSome and EncryptAll, each with the trivial trap comprising all states of the phase; furthermore, both traps triv are connecting to the other phase. Partition LoPo and role PDA(LoPo) at its level are depicted in Figure 3.

(a) EncryptSome

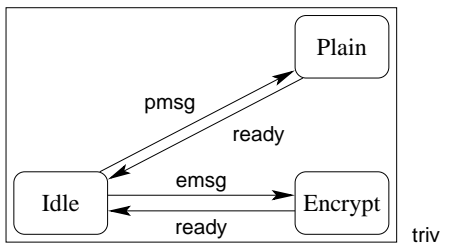

(b) EncryptAll

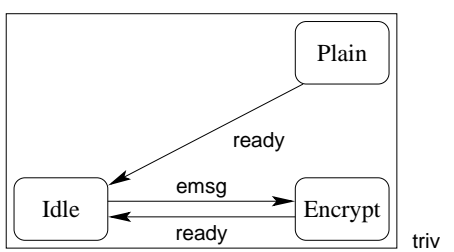

(c)
EncryptSome

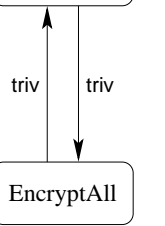

Fig. 3. (a-b) Phases and traps, (c) corresponding role PDA(LoPo)

To complete the picture, the overall collaboration involving the two protocols InOrOut and PlainOrEncrypt of PDA, email client and security module, is drawn 
in Figure 4. In InOrOut, the security module conducts PDA in its role PDA(LoPo); in the PlainOrEncrypt protocol, PDA conducts the email client in its role EMC(DoPo). In the figure conducting is indicated by thin boxes.

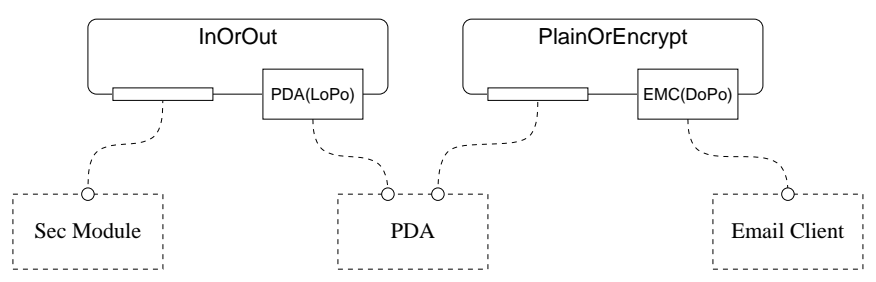

Fig. 4. Collaboration: protocols InOrOut and PlainOrEncrypt.

Clearly, the above InOrOut protocol does not model the possibility for the PDA user to override the location security policy. As depicted in Figure 5, we extend the detailed STD of PDA with a new state Override and two transitions (a), and we add a new phase (d) to PDA's role LoPo (e). Furthermore, we redefine the original two phases, in view of the addition of state Override $(\mathrm{b}, \mathrm{c})$. The protocol InOrOut is extended with two consistency rules as choreography steps. Also, a consistency rule as orchestration step is added, dealing with the mobility of PDA conducted by security module SM.

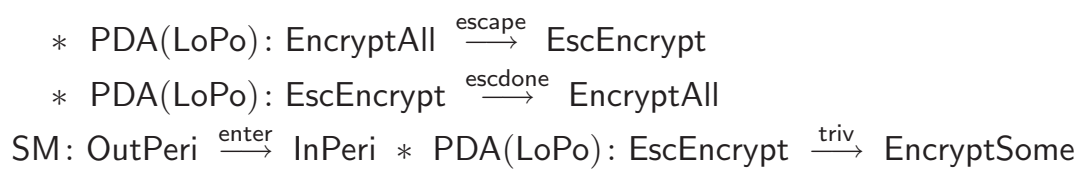

In the first choreography step, the PDA, once in the trap escape, can transfer unconductedly to phase EscEncrypt in which a pmsg-transition to the state Plain is available. The second choreography step transfers the PDA unconductedly to phase EncryptAll once trap escDone has been entered. However, to assure no restrictions apply any longer in case the PDA has returned into the security perimeter while sending the message, the consistency rule conducted by SM is added.

As can be seen from Figure 5c for phase EncryptAll, the inner trap escape contains the new state Override. The trap escape is used to catch the PDA user's wish to override the standard encryption regulation. The outer trap triv is still needed, viz. for the former consistency rule transferring the PDA to phase EncryptSome. The new phase EscEncrypt in the role LoPo of the PDA, Figure 5d, has a transition labelled pmsg to the state Plain. Note, neither phase EncryptAll nor EscEncrypt have a pmsg-transition leaving from state Idle. Only in the special case of overriding, the sending of plain messages is allowed. In order for the email client to stay consistent with this transition, the consistency rule

$$
\text { PDA: Override } \stackrel{\text { pmsg }}{\longrightarrow} \text { Plain } * \text { EMC }(\text { DoPo }): \text { Finished } \stackrel{\text { free }}{\longrightarrow} \text { PSend }
$$


(a)

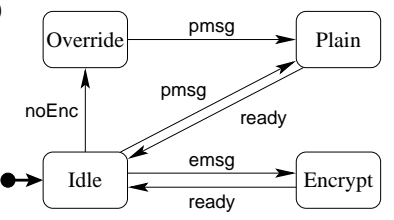

(e)

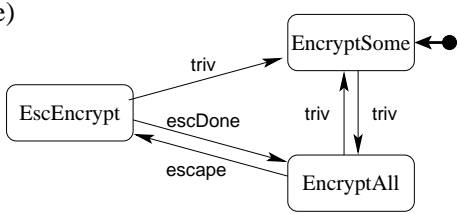

(b) EncryptSome

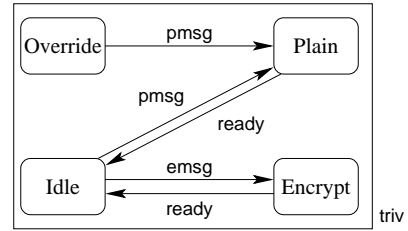

(c) EncryptAll

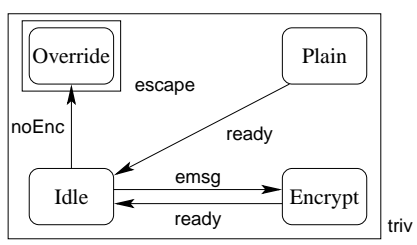

(d) EscEncrypt

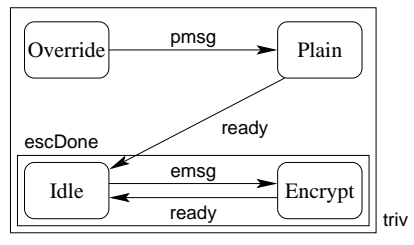

Fig. 5. Overriding: (a) adapted STD for PDA, (b-d) phases, (e) role PDA(LoPo).

is to be added to the PlainOrEncrypt protocol. Note, the STD of the email client itself is not changed. The overriding of the location security policy ends once trap escDone of phase EscEncrypt is reached, a signal caught by the second choreography rule above.

Using the example of security policies regulating plain or encrypted sending of email, we have illustrated Paradigm's key notions of STD, phase, (connecting) trap, role and consistency rule as well as the terminology of protocol, orchestration and choreography. In the next section, we shall exploit Paradigm notions and terminology in describing the well-known FOO voting scheme.

\section{A voting example: architecting interaction}

In this section we address a substantially larger security protocol, the so-called FOO voting scheme proposed by Fujioka, Okamoto and Ohta [14]. The example is small enough for the size of this chapter, but also large enough to underpin our architectural ideas concerning security systems. It comes down to the following. We take a security problem as an interaction situation, where specific interactions are controlled via dynamic constraint regulations. This is modeled within Paradigm via suitable groupings of collaborating components into UMLlike collaborations, each responsible for a certain aspect of the overall interaction. Each collaboration then can be taken as a separate architectural unit of a security concern, to be analyzed and understood in relative isolation, resulting in a dedicated specification of a solution for that concern. Via the consistency inherently provided by Paradigm, well-separated concern solutions can be re-united and integrated into a complete solution for the security situation. Based on the architectural organization into separate concerns, the complete solution can be overseen and remains manageable in terms of partial solutions. 
For the purpose of this paper, an abstract description of the FOO voting scheme suffices. See $[25,34]$ for more details. The scheme distinguishes between three main stages. As a first step, the Organizer of the election makes public that an election will take place. During the first stage of the election process, Humans register with the so-called Administrator. During this stage, the Human contacts the Administrator, identifies himself and sends a blinded message containing his encrypted vote to the Administrator. Due to the blinding, the Administrator cannot determine the message of the Human. In case the Human is entitled to vote, the Administrator will sign the Human's blinded message and return it. At a certain moment the first stage will end, meaning that Humans entitled cannot obtain the possibility to vote any more.

During the second stage a Human can send his encrypted vote signed by the Administrator to a so-called anonymous channel. This Channel collects all received encrypted votes and signatures and sends these to the Counter in an arbitrary order. For simplicity, we assume that Channel first collects all encrypted votes and then forwards them in bulk. Thus, the output message of the Channel cannot be related to a specific Human, providing anonymity of votes. The Channel does not check the correctness of the messages it obtains from the Humans, it only reorders messages. The second stage, encrypted voting, needs to take place within a certain period specified in advance: Humans wanting to cast their votes, need to send their encrypted votes to the Channel in time.

During the third stage, each voter uncovers his vote anonymously. To that aim, each voter sends his uncovering, i.e. the key he used for encrypting his vote, to the Counter via the Channel. As the output of the Channel hides the sender of the output message, the privacy is protected. Each voter in the scheme makes use of the Channel twice. First, the Channel collects all encrypted votes. After this, the Channel outputs them in an arbitrary order. Analogously, the anonymous channel first collects all keys for uncovering and after that outputs these to the Counter in arbitrary order. The set-up, according to the voting scheme, guarantees full anonymity by strict separation of subsequent stages: (i) administering and encrypted voting, (ii) first bulk output, (iii) uncovering, (iv) second bulk output and counting.

As we see from the description, the FOO voting scheme has five different types of components: Human, Organizer, Administrator, Channel and Counter. The number of the Human components is undetermined, $n \in \mathbb{N}$ say. Of the remaining four types there is exactly one of each. Given the above, we differentiate between two major activities: ElectionOrganizing covering the overall voting procedure; VoteHandling covering the individual handling of voters and votes.

Figure 6 presents the roles of each component grouped into two separate collaborations. No role is given for Organizer, as he only conducts the protocol of collaboration ElectionOrganizing, as indicated by the thin unlabeled box. Indeed, ElectionOrganizing will be coordinated by orchestration. Each Human ${ }_{i}$, $1 \leq i \leq n$, participates in ElectionOrganizing and in VoteHandling in its two roles Human $_{i}\left(\right.$ InElection) and Human ${ }_{i}$ (AsVoter), for brevity written as Hum ${ }_{i}(\operatorname{InElec})$ 


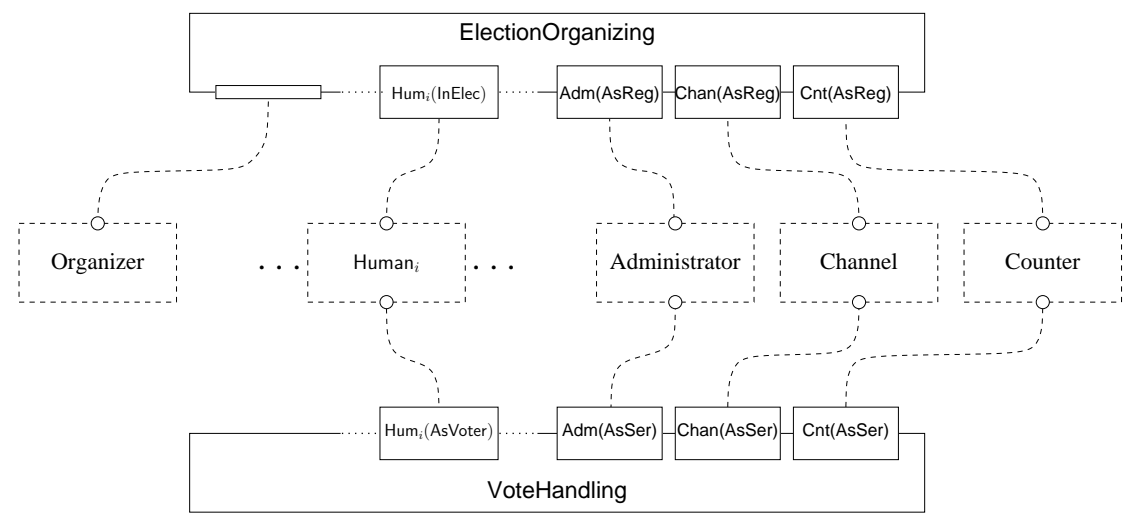

Fig. 6. Collaborations ElectionOrganizing and VoteHandling.

and $\mathrm{Hum}_{i}$ (AsVoter), respectively. Using the shorter names Adm, Chan and Cnt for the Administrator, Channel and Counter, their cooperation within the collaborations is via their roles AsRegulator and AsServer, written as AsReg and AsSer in the figure. Note, the protocol of collaboration VoteHandling has no conductor; no thin unlabeled box is present. The protocol will be coordinated choreographically. Except for Organizer, all components are contributing to both protocols, but via two different roles, one for each protocol exclusively. Components do not belong themselves to a collaboration, but their roles do so instead. In view thereof the components are visualized in dotted form.

We proceed by explaining the dynamics of the orchestration of the collaboration ElectionOrganizing. Because of its overall guiding character, it is easier to explain than the choreographic VoteHandling. The dynamics of the latter will be addressed thereafter.

In its five parts, Figure 7 visualizes the detailed STDs of the five components. Organizer clearly takes four fixed consecutive actions. The first action announce allows all Human components to perform their first two main voting activities of administering and encrypted voting. The second action start allows the Administrator to become active. From then, Humans can be handled by Administrator and subsequently by Channel. The third action proceed is done only after the encrypted votes have been received by the Counter. The fourth action declare is done after all vote uncoverings have been processed and counted. Only then the result of the election is made known.

Any Human can try to behave as a voter by doing the eight actions leading from starting state Idle to state Voted. In state Idle, after having heard he may or may not vote, such a Human can choose to do action hear or no-Hear. Similarly, in state Invited, he may choose to do action askForm or no-Ask. In state ToForm he does action getForm if and only if Administrator allows so. In state WithForm he can choose action complete or no-Complete. In state Filled, while being served by Channel, he does action sendEnc. In state EncSent he chooses to do action waitUncover or no-Wait. In state Waiting, he can choose to wait until it is allowed 


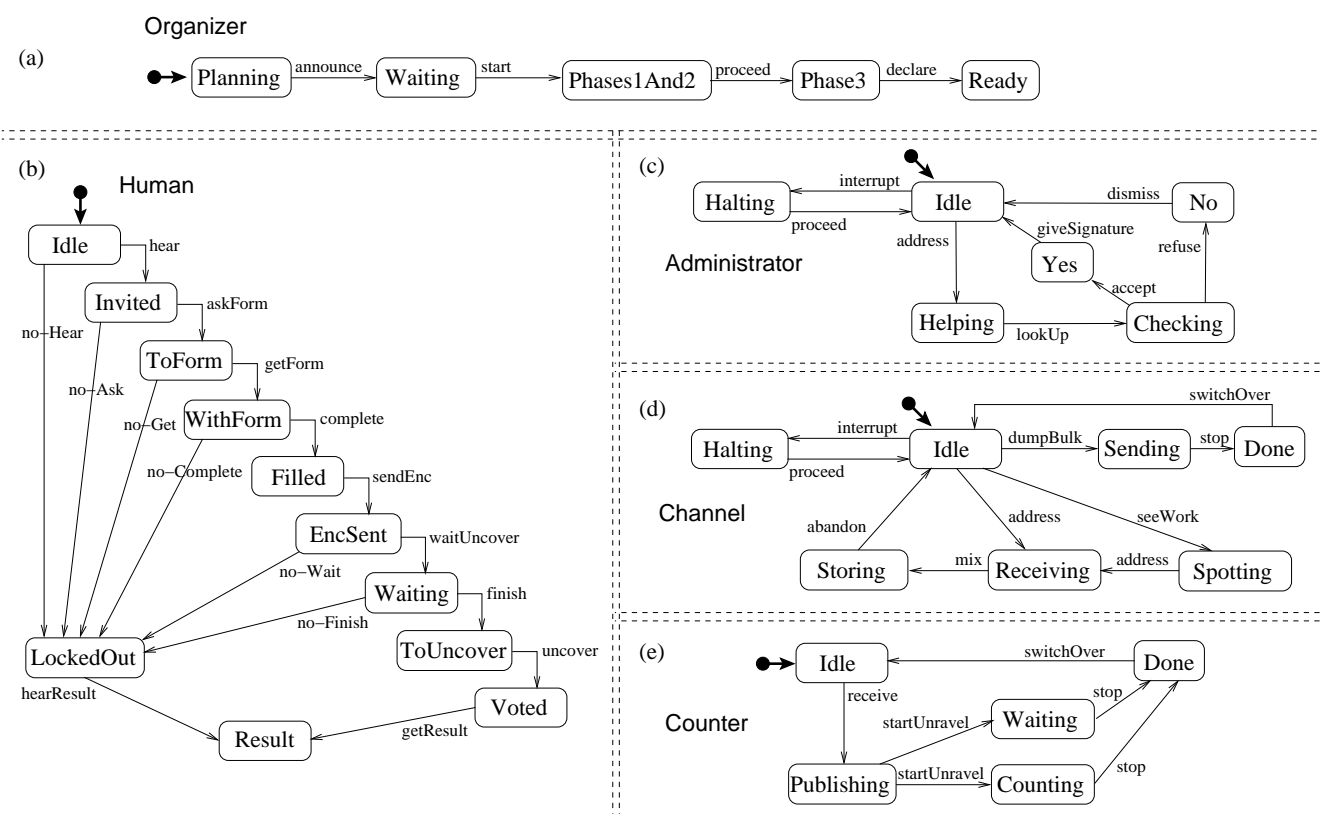

Fig. 7. Detailed STDs: Organizer, Human Administrator, Channel, Counter.

to uncover by doing action finish, or he can quit earlier by doing action no-Finish. In state ToUncover, while again being served by Channel, he does action uncover. In state Voted, he waits until the election outcome is made known, upon which he does action getResult. Similarly, in state LockedOut, he waits until the election outcome is made known, upon which he does action hearResult.

Figure 7c presents Administrator. Starting from state Idle, any Human asking for a form, can be handled individually by Administrator as follows. First he does action address, then action lookUp for a particular Human. Depending on the result thereof, he either continues by doing action accept followed by giveSignature, or he continues by doing refuse followed by dismiss. After both continuations, Administrator is back in state Idle, ready to handle another Human. In addition, Administrator has another possibility in Idle as a kind of closing-time policy: first doing action interrupt followed by doing action proceed, thus returning to Idle once more. This action pair is done exactly once, as we shall see. Upon returning to Idle each Human not yet having asked for a form, cannot do so any longer, but all those that already did, will be handled, one after another indeed.

Channel takes over individual Human handling from Administrator. Moreover, it actually does so two times, first for the encrypted votes they cast. Channel explicitly closes encrypted vote handling, for every Human involved, by going to state Done for the first time. After that, it continues Human handling for their uncoverings. Again, it explicitly closes this handling by going to state Done for the second time. Starting from state Idle, each time a Human turns up, Channel does action address, thus receiving the encrypted vote. Then it does 
action mix, scheduling the encrypted vote to be delivered later. Finally it does action abandon, thus ending service to the particular Human and returning to state Idle.

Like Administrator in state Idle, Channel has, as closing-time policy, the possibility of doing action interrupt followed by proceed, thus returning to Idle once more. Upon returning to Idle each Human not yet having turned up, cannot do so any longer, but all those that already did, will be served, one after another indeed. To that aim, action seeWork leading to state Spotting is chosen to address any such waiting Human individually, whereupon actions address, mix and abandon lead back to Idle. As soon as no other Human is waiting to be served, it takes action dumpBulk, thereby forwarding all encrypted votes together to Counter for further handling. The action stop leads to state Done. By taking action switchOver returning to state Idle occurs. Subsequently, Channel displays highly similar behaviour once more, now for handling any Human's uncovering in the same manner as it handled his encrypted vote.

Like Channel, Counter as given in Figure 7e is involved twice in vote handling: first, receiving all encrypted votes together and publishing them, be it still encrypted, and, second, receiving all uncoverings together and applying these, thereby doing vote counting too. Starting from state Idle, Counter takes action receive, thus receiving all encrypted votes as one bulk. Then, by taking action startUnravel, it goes to state Waiting, where all encrypted votes are being published. To finish encrypted vote handling, Counter takes action stop towards state Done. By taking step switchOver it returns to Idle from where it repeats the three actions receive, startUnravel and stop returning to state Done again, but in this case via state Counting. By doing so, it first receives the uncoverings, it then processes all uncoverings received, thereby counting the votes too. After all uncoverings have been applied and the votes have been counted, it stops in Done at last.

The STDs discussed above are detailed STDs. Only one of these, Organizer, participates as such in collaboration ElectionOrganizing. The other four STDs do participate, but more indirectly, via their respective roles InElection and AsRegulator, see Figure 6. Each such role is a global STD, whose states and actions are phases and connecting traps, respectively, from a particular partition. These partitions as well as the roles built from them, are visualized in Figure 8 for Human and in Figure 9 for Administrator, Channel and Counter.

Note how the four phases of partition InElection of the role Human(InElection), subsequently allow a Human more freedom: first it can do nothing in phase PreElection, second it can go as far as sending its encrypted vote in phase HalfWayVoting, third it can even do the uncovering in phase FinalVoting, and last it can hear the outcome of the voting in phase Outcome. Trivial traps here facilitate phase transfers independently from Human behaviour within a phase.

Figure 9a depicts how Administrator first can do nothing in phase Passive and, second, can do things unrestrictedly in phase Active. Slightly less simple, Figure 9b expresses how Channel, in phases Active1 and Active2, can do things 


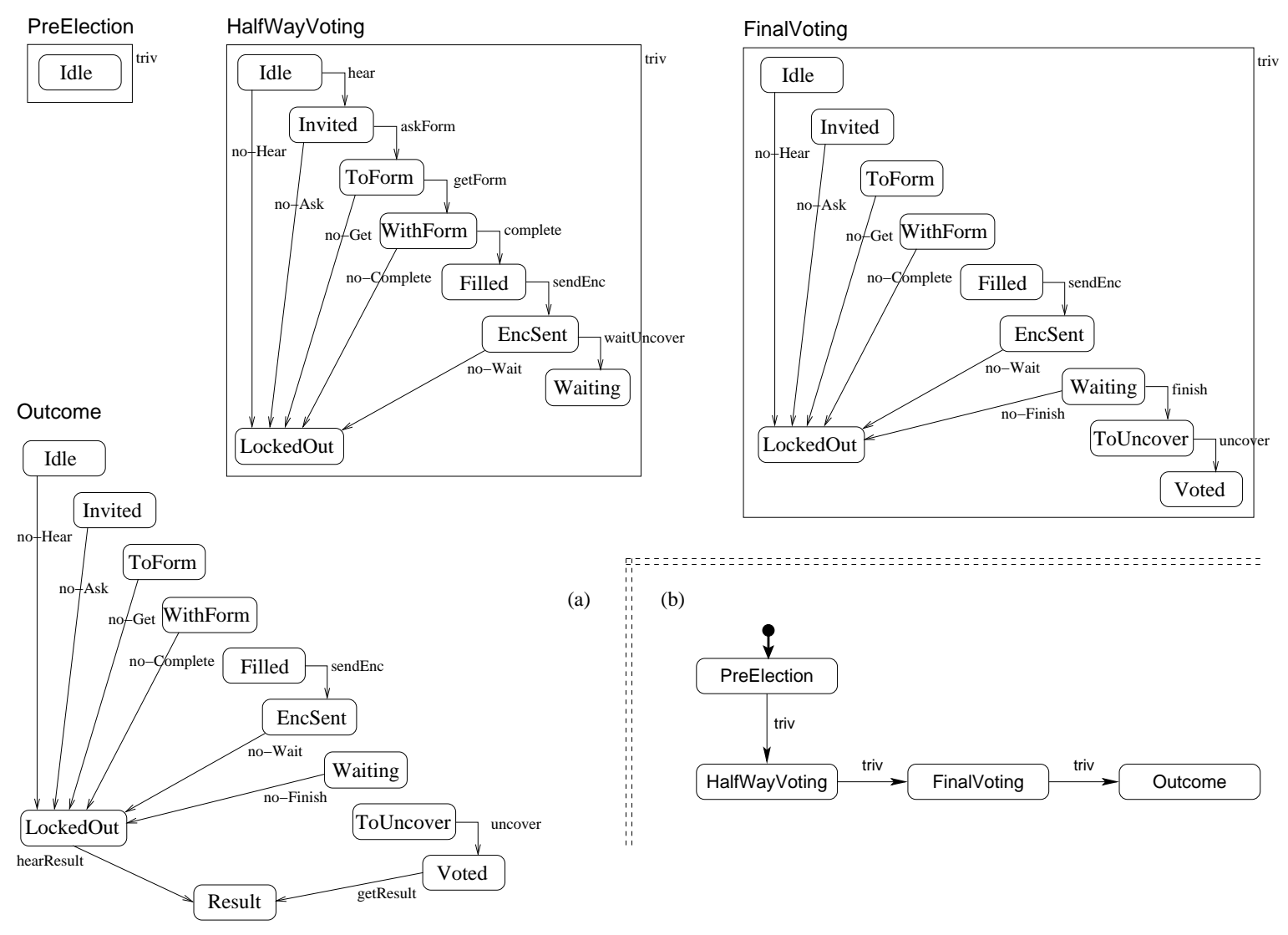

Fig. 8. (a) Partition InElection of $\operatorname{Human}_{i}$, (b) corresponding role Human ${ }_{i}\left(\operatorname{InElection}_{\text {). }}\right.$

unrestrictedly between state Idle and state Done. Phase Active1 covers all encrypted vote handling by Channel, phase Active2 covers all uncovering handling by Channel. Their difference is, in phase Active2 component Channel can no longer resume its activities after having arrived in state Done.

Figure 9c expresses similar phases for Counter, where phase ShowingEncs is the analogue of Active1 above, precisely during phase HalfWayVoting of all Humans together. Similarly, phase ShowingVotes is the analogue of Active 2 above too, but in this case during phase FinalVoting of all Human together, where vote uncoverings are being published and counted accordingly. The phase Resumption bridges the behavioral gap between the two.

It is via these coarse-grained constraints, Organizer conducts the election procedure. By action announce he unleashes all Humans into phase HalfWayVoting while still keeping Administrator in phase Passive. It is only by Organizer's second action start, he puts Administrator into phase Active. By his third action proceed, on the basis of Counter having closed encrypted vote handling, Organizer conducts all Humans into the third phase FinalVoting. In addition, both Channel and Counter are conducted to their next phases, Active2 and Resumption, respectively. 
(a)

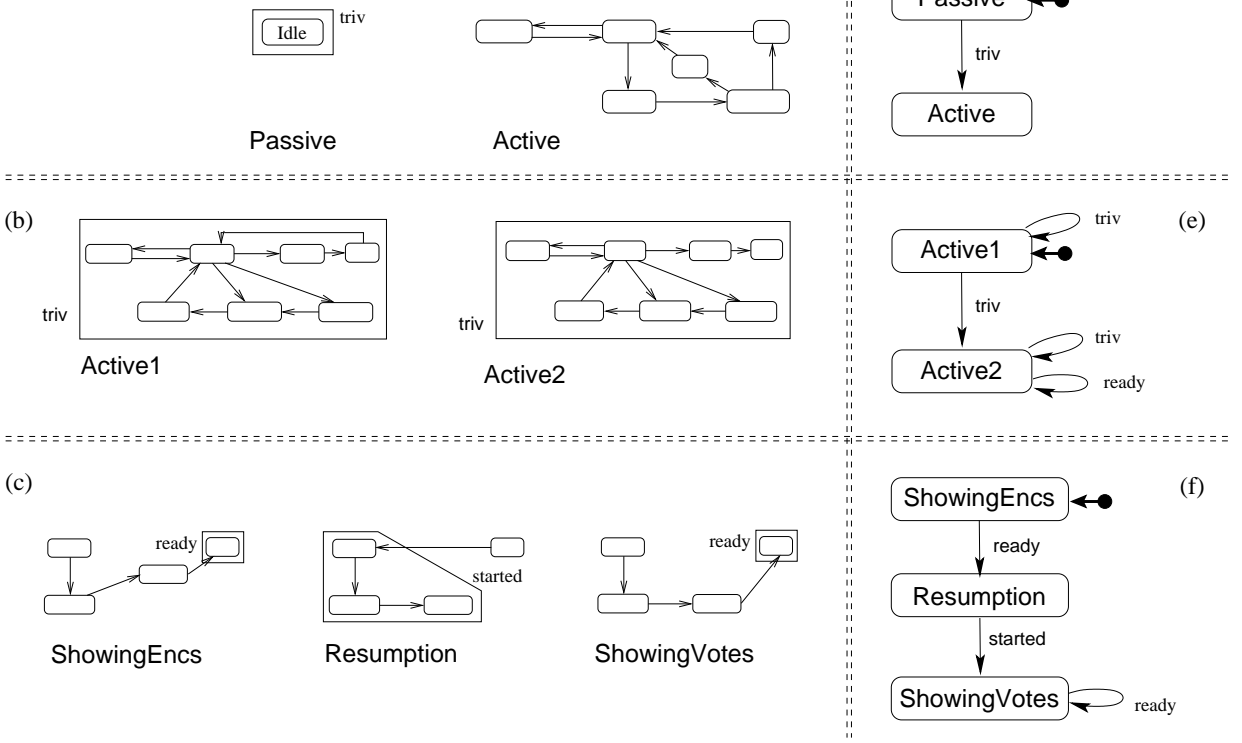

Fig. 9. (a-c) Partitions AsRegulator of Administrator, Channel and Counter, (d-f) corresponding roles.

Note, this is to happen only, if both Counter in its role AsRegulator and Channel in its other role AsServer have made enough progress. The combined condition for such progress is: trap ready of phase ShowingEncs (for Counter) as well as trap ready of phase Finishing (for Channel) are the two relevant, nontrivial traps currently entered. In the case of the latter trap ready, its having been entered should be observed only, since phase Finishing is not to be transferred -such dynamics belong to Channel's role AsServer in other protocol VoteHandling. Finally, on the basis of Counter having closed vote uncovering, Organizer takes his last action declare, conducting all Humans into the last phase Outcome. The consistency rules specify this precisely. From their format one can directly recognize the orchestration character of the protocol of collaboration ElectionOrganizing. Note, the use of the universal quantifier to abbreviate the notation in three of the rules, thus establishing a broadcast to each Human $_{i}$ via his InElection role: one synchronized role step for all $n$ Humans together.

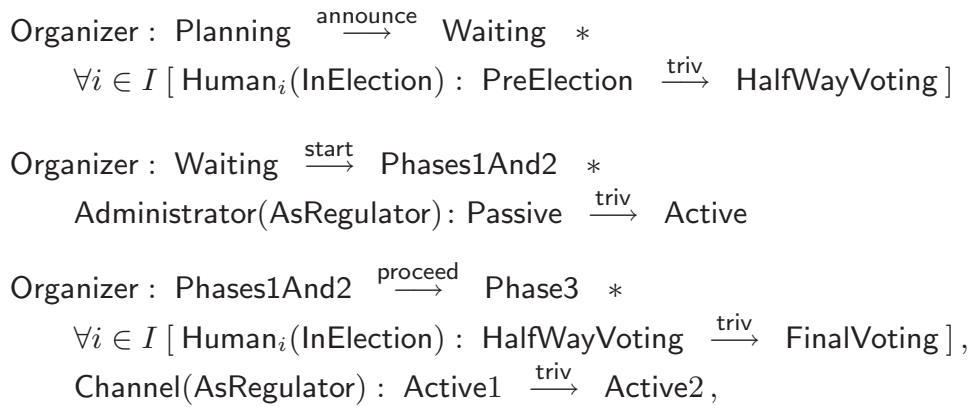




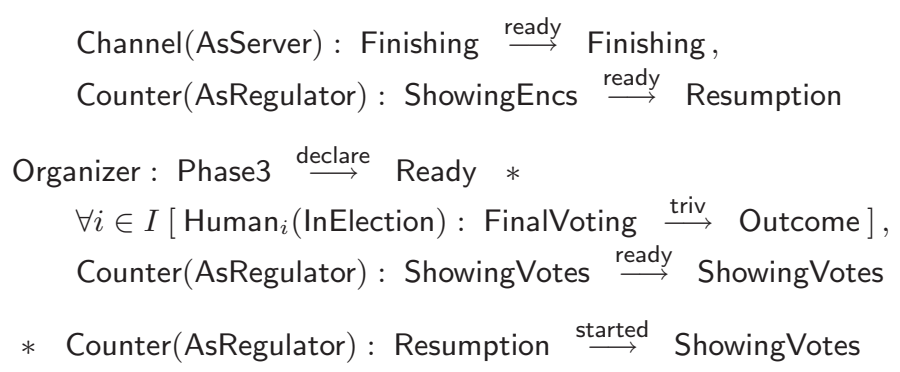

The last consistency rule is a choreography step assuring, Counter swaps from phase Resumption to phase ShowingVotes after trap started has been entered. This concludes the coordination of collaboration ElectionOrganizing addressing the overall conducting.
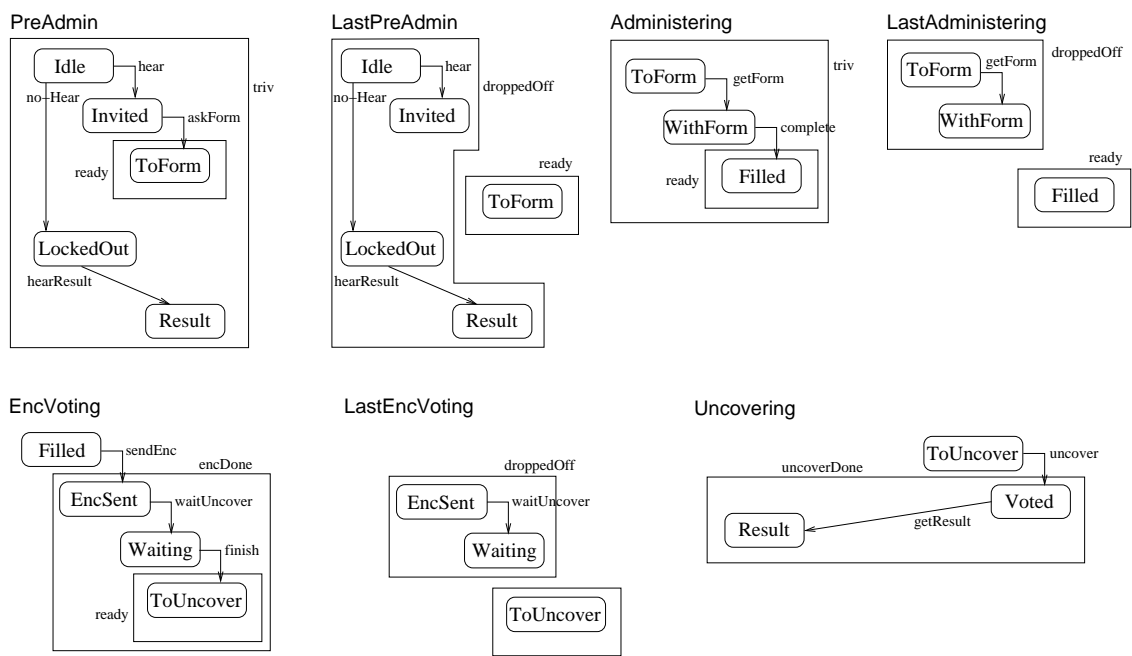

LastEncVoting

Uncovering
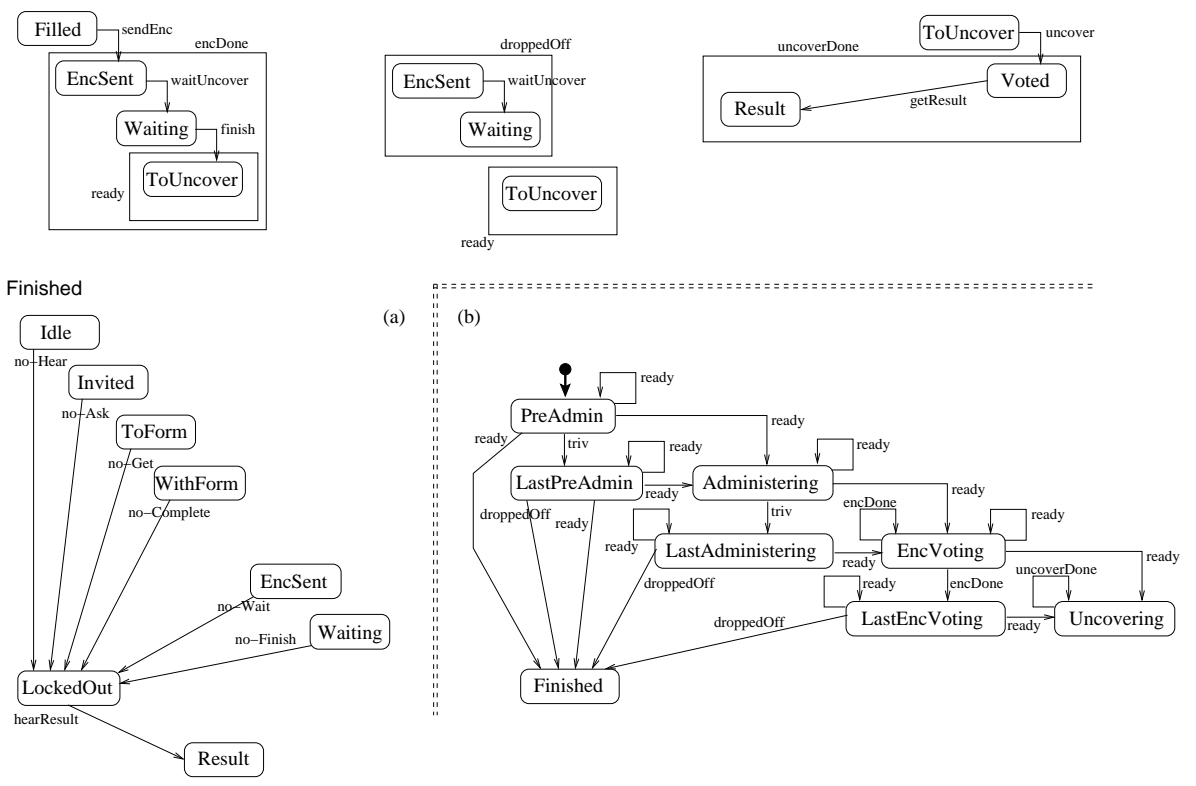

Fig. 10. (a) Partition AsVoter of Human, (b) role Human(AsVoter). 
For the remainder of this section we direct our attention towards the collaboration VoteHandling. Figure 10a gives partition AsVoter of Human, Figure 10b gives the corresponding role. We briefly discuss phases, traps and role, first by concentrating on successful, normal voting. Four phases have been defined: PreAdmin, Administering, EncVoting and Uncovering. First, phase PreAdmin where the role starts by allowing any Human to ask for the signature providing service of Administrator; second, phase Administering where the signed form is given to the voter; third, phase EncVoting where encrypted voting is done and a voter can ask to uncover his vote; fourth and last, phase Uncovering where uncovering of a voter's encrypted vote is done.

Note, within phase PreAdmin any human indeed has the choice between trying to get a signed voting form and not trying to, independent from having the right to vote. So, a prohibiting outcome, different from getting the normal next phase Administering imposed, should be possible, even if trap ready within phase PreAdmin has been entered. The prohibiting outcome is represented by phase Finished. As we shall specify below, this is handled by Administrator via its role AsRegulator. Thus, Administrator is involved in any Human's phase transfer from PreAdmin either to Administering or to Finished, both via connecting trap ready. In relation to the other normal phase transfers, we have omitted the prohibiting outcome as a possibility, for space reasons only. Thus, similar but simpler, Channel is involved in any Human's phase transfer from Administering to EncVoting and, again, Channel is involved in any Human's phase transfer from EncVoting to Uncovering. So, two components are pipeline-wise involved in the three subsequent, successful, normal phase transfers of any Human.

However, there is some time pressure too. So, on the basis of trap triv of phase PreAdmin, or of Administering or on the basis of trap encDone having been entered of phase EncVoting, the particular phase can be interrupted. In case a Human turns out to have entered trap droppedOff instead of trap ready, the responsible component is not going to serve that Human and the Human transfers to Finished, as he was too late in asking for the next service needed.

According to the above explanation, the non-Human component Counter is not responsible for any Human's phase transfers. The reason is, Counter cooperates with Channel exclusively and only twice. The first time is, the sending of all encrypted votes together; the second time is, the sending of all uncoverings together. We shall clarify this point later, after first having explained the phases of both Administrator and Channel relevant for the pipeline-wise guiding of each Human component through his role AsVoter. To that aim we refer to Figures 11 and 12. They visualize the phases from partition AsServer of the two components Administrator and Channel, together with the corresponding roles.

In Figure 11, we depict how Administrator starts in phase Finishing, where it finishes the signature providing service to a Human by entering trap ready and where it cannot start a next service. In phase Handling ${ }_{i}, 1 \leq i \leq n$, it can start doing so for $\operatorname{Human}_{i}$ only, as follows from the consistency rules below. Serving proceeds up to either giving the signature or refusing to give it: by entering trap signature or trap noSignature respectively. Via these traps it returns to phase 


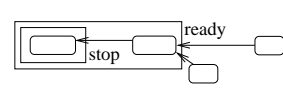

Finishing

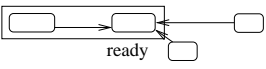

LastFinishing

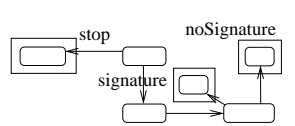

Handling $_{i}$

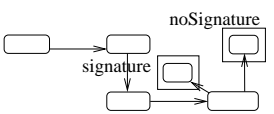

LastHandling

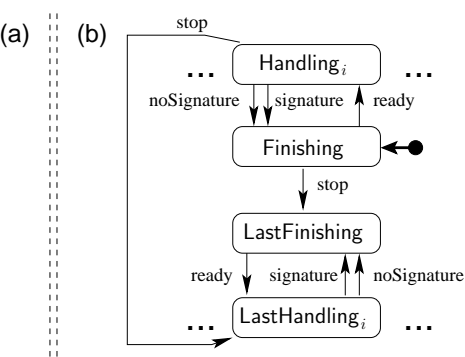

Fig. 11. (a) Partition AsServer of Administrator, (b) role Administrator(AsServer).

Finishing, where it will get ready for handling another Human asking for the signature. In all $n+1$ phases discussed so-far it has the additional possibility to enter trap stop, which it does at closing time. From stop in Finishing, it continues in LastFinishing and from stop in Handling ${ }_{i}$ it continues in LastHandling ${ }_{i}$. Phase transfers between LastFinishing and LastHandling ${ }_{i}$ are exactly similar as discussed above between Finishing and Handling ${ }_{i}$. The difference is in the Human components, however. From now on no new Human components can start asking for the signature providing service. This means, only those who were already asking for it before trap stop was entered by Administrator, have to be served. Below, consistency rules specify this, serving Humans one by one.

Figure 12 visualizes similar phases and role of Channel in view of providing to a Human the service of anonymously sending his encrypted vote or his uncovering thereof. The role starts in phase Finishing, where it waits inside trap ready to start a new service turn. Until further notice, this service is the anonymous bulk sending of encrypted votes only. After having been asked by Human ${ }_{i}$, it provides a fresh service turn, exclusively to Human ${ }_{i}$, by going to phase Handling ${ }_{i}$ where it enters trap next, via which it returns to phase Finishing. Similar as above, the additional trap stop is used in view of the closing time policy. Via trap stop a swap is made to phase LastFinishing or to phase LastHandling ${ }_{i}$. Via these two phases, any Human that had asked for the anonymous encrypted vote sending service in time, is being served. Only then, all encrypted votes together are being sent to Counter. It is via trap bulkSent of LastFinishing the role returns to Finishing, where it restarts providing the anonymous sending service to any Human asking for it, this time with respect to vote uncoverings. So it returns to phase Finishing where it can reenter trap ready soon enough. This is particularly relevant for the last consistency rule of the ElectionOrganizing protocol starting on page 15 .

The roles given in Figures 10, 11, 12, are synchronized through the following consistency rules. Their synchronization constitutes the main part of the protocol for collaboration VoteHandling; the remaining part will be discussed separately, through the additional AsServer role of Counter. Note, none of the protocol steps, being the consistency rules, has a conductor, in line with the choreographic character of the protocol. To facilitate the discussion, we split the presentation of the rules into three groups. 


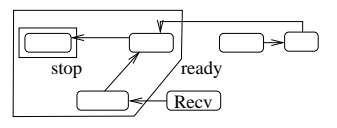

Finishing

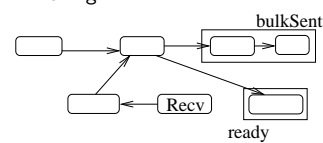

LastFinishing

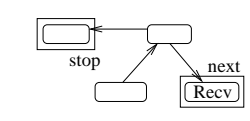

Handling $_{i}$

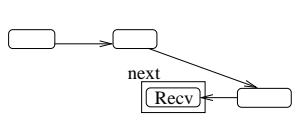

LastHandling $_{i}$ (a)

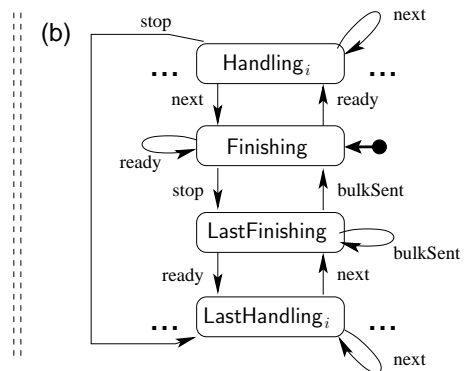

Fig. 12. (a) Partition AsServer of Channel, (b) role Channel(AsServer).

The first group of eight rules specifies how Administrator serves a single Human $_{i}$ in his role AsVoter, thereby transferring him from phase PreAdmin either to Administering or to Finishing, possibly via LastPreAdmin in view of the closing time policy. The first three rules of the group address how a Human $i$ in two steps is being transferred from PreAdmin to Administering or to Finished. The last three rules address a similar transfer from LastPreAdmin. The two middle rules address phase transfers needed for the closing time policy, transferring all relevant Humans together from PreAdmin to LastPreAdmin. Note, the universal quantifier ranges over those Humans still being in PreAdmin.

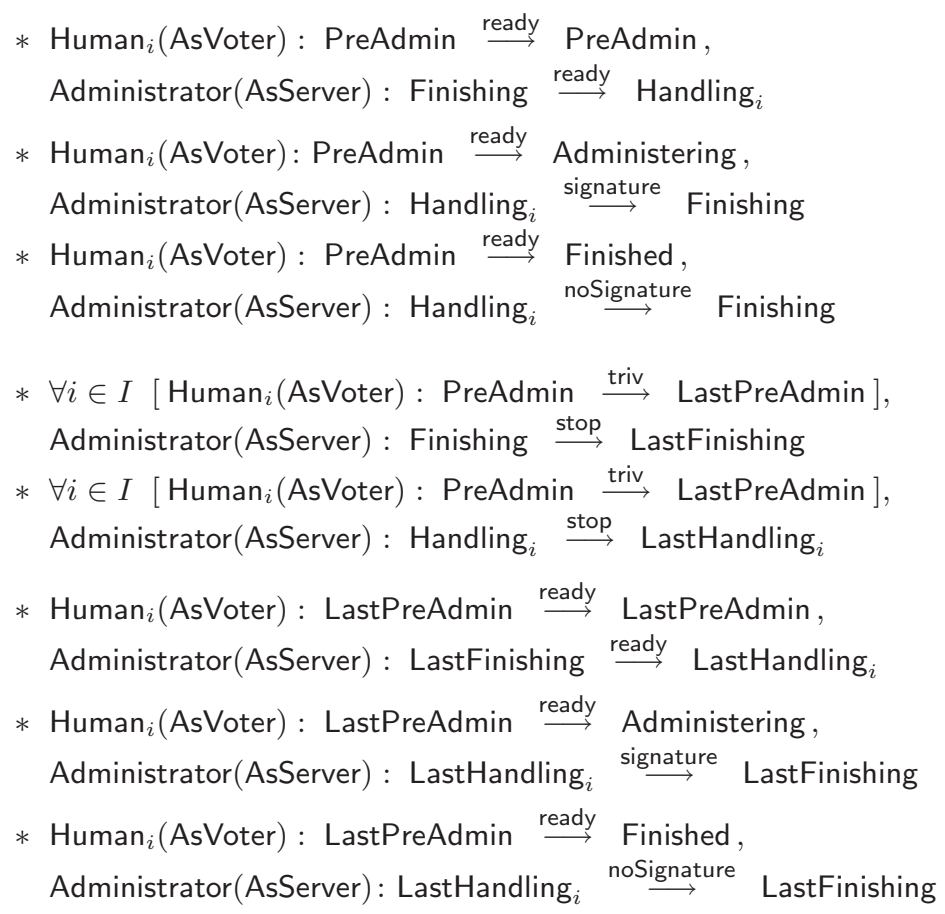

The above rules do not express, what happens to a Human trapped in droppedOff of phase LastPreAdmin. This is covered by the second group of three consistency rules, additionally expressing what happens to a Human similarly trapped in droppedOff of phase LastAdministering or of phase LastEncVoting. Note, in these 


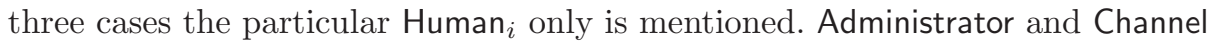
are not involved.

* Human $($ AsVoter $):$ LastPreAdmin $\stackrel{\text { droppedOff }}{\longrightarrow}$ Finished

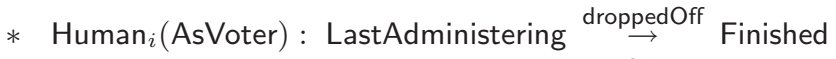

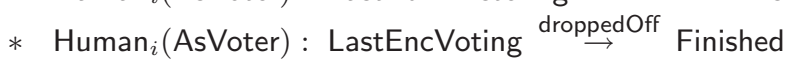

The third group of rules is resembling the first group, specifying how Channel serves a particular Human $i$ in his role AsVoter. Thereby, Channel firstly transfers him from phase Administering to EncVoting and secondly transfers him, much later, from EncVoting to Uncovering. In view of closing time policy, the first transfer may take place via LastAdministering and the second transfer via LastEncVoting. As we did not take into account the possibility of a refusal, the rules are more simple in that respect than those from the first group. A slightly less simple detail, however, arises in the service offering by Channel when in trap next. Channel has to remain inside phase Handling ${ }_{i}$ until it indeed has received the encrypted vote from $\operatorname{Human}_{i}$, before it can schedule the vote for output. The same detail is observable in other rules as well. Again, the closing time policy is addressed. Here, we need an additional detail concerning discriminating between handling encrypted votes and handling uncoverings. So, an inspection of its own current phase in the other role AsRegulator has been added to the rules, about being in trap triv either of phase Active1 or of phase Active2.

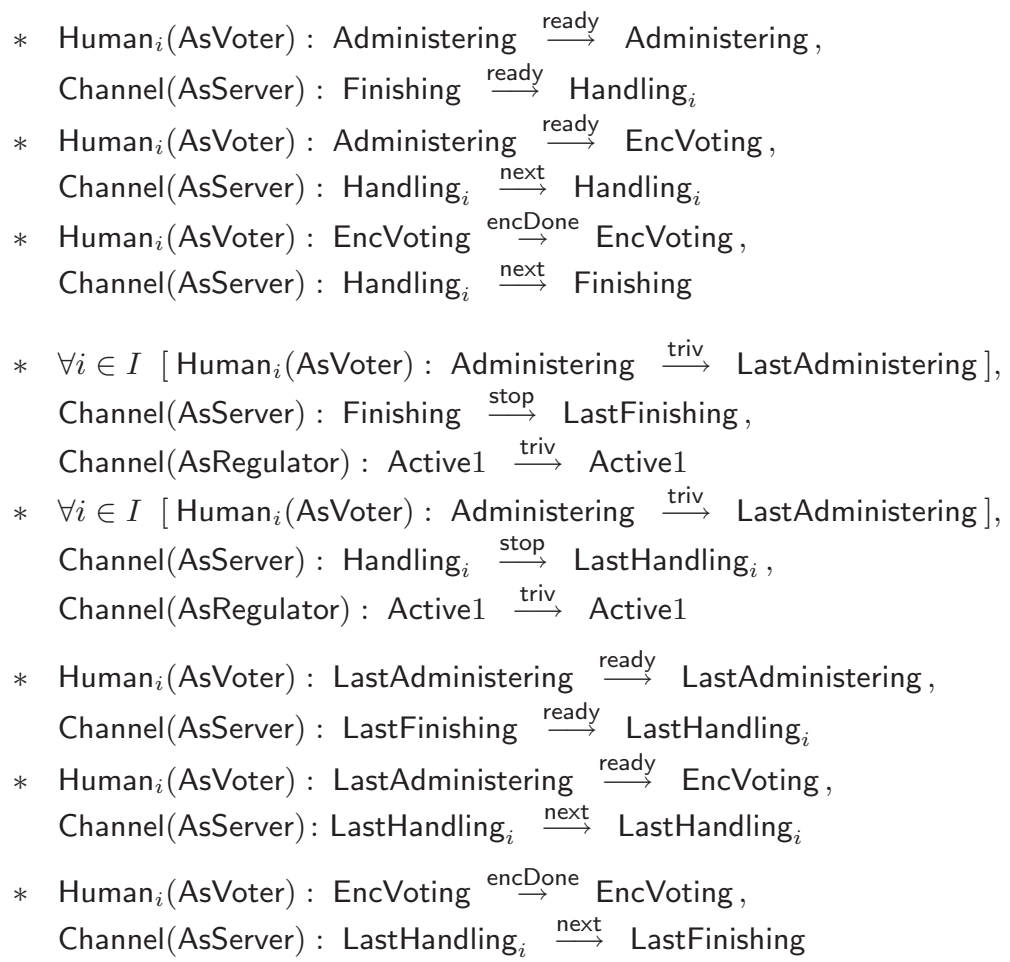




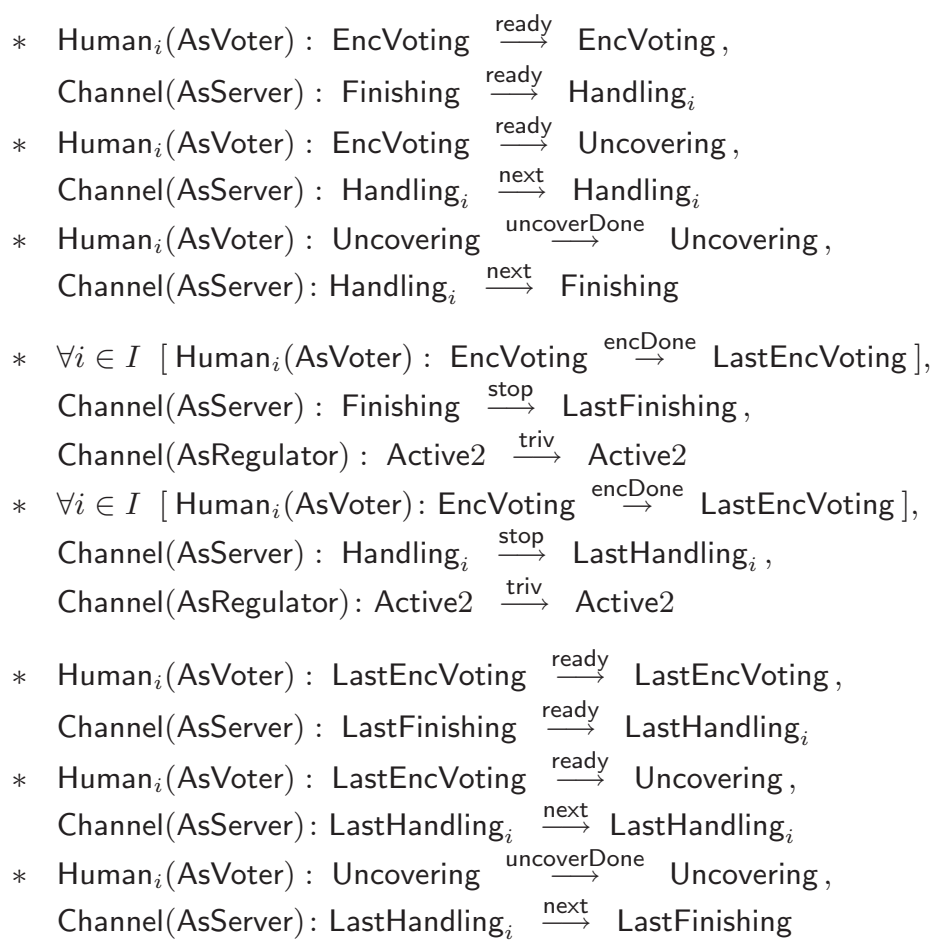

So far we have discussed the consistency rules coupling the AsVoter role of Human and AsServer roles of Administrator and of Channel. In the remainder of this section we explain the AsServer role of Counter and how it is coupled to the AsServer role of Channel. Figure 13 presents partition AsServer of Counter and the corresponding role. The role starts in phase Finishing, where in trap ready it is waiting for the first bulk to arrive. Such a bulk gets handled in phase Beginning, where via the large trap started the role returns to phase Finishing, but the actual handling newly started just continues within Finishing to its regular end.

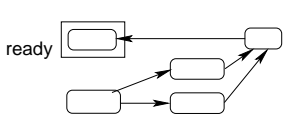

Finishing

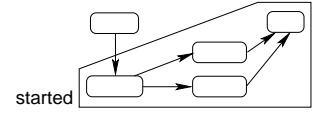

Beginning

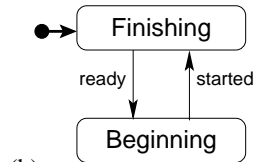

(a) $\|($ (b)

Fig. 13. (a) Partition AsServer of Counter, (b) role Counter(AsServer).

The consistency rules coupling the AsServer role of Counter from Figure 13b and the AsServer role of Channel from Figure 12d are the fourth and last group of rules specifying the protocol for collaboration VoteHandling. It has two rules only. The first rule couples trap bulkSent of Channel having been entered in its phase LastFinishing, to a transfer to phase Beginning of Counter. Thus the end of Channel's service providing during either all Humans' simultaneous phase HalfWayVoting or their simultaneous phase FinalVoting has been reached. Hence, 
encrypted vote publishing or uncovering results are to be initiated by Counter, uncovering combined with the counting the votes. Recall, the actual restart of Counter is done by Organizer in protocol ElectionOrganizing.

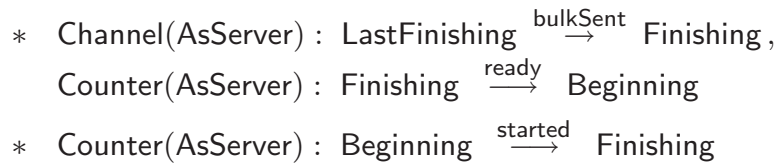

As a final remark we like to observe, it is the clear separation of two concerns, achieved through the two collaborations chosen, which has been instrumental in constructing the Paradigm model and in subsequently explaining it. One concern is the overall voting procedure for all Human components together, the other

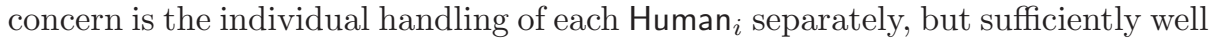
in line with the overall procedure. Additional features then turned up, like closing time, which we incorporated completely, and like malicious behaviour, which we addressed superficially only, via the two possible outcomes of the service provided on an individual basis by Administrator. How to handle truly malicious and unintended behavior is a topic of ongoing research however. This is also relevant outside the field of security; e.g. in business process modeling and in computer supported collaborative work.

\section{Model checking safety and security properties}

General Paradigm models, in particular security architecture models as above, can be translated into the process language of the model checker mCRL2 [22,21]. This way one can formally verify whether an architectural description satisfies certain requirements or whether it exhibits specific undesired behaviour.

Characteristic for mCRL2 are the support for abstract data types and the use of parametrized boolean equation systems for symbolic model checking [23]. A process description $P$ and the property $\varphi$ to be checked together yield such an equation system. Solving the equation system provides the answer whether system $P$ satisfies property $\varphi$. In addition, mCRL2 provides extensive support, e.g. for the generation and visualization of LTSs. ${ }^{3}$

In outline, the translation of Paradigm into mCRL2 is as follows. A component is represented by the parallel composition of its detailed STD and all its roles. It expresses the component's current local state as well as the current phases for all its roles. Within the parallel construct, state information is communicated from the detailed STD to the global ones, allowing them to update their trap information. Vice versa, according to the Paradigm semantics, a transition to be taken at the detailed level requires the transition to be allowed by all the phases the component is currently in. By a proper synchronization of the actions involved in state updates and transition requests, consistency between detailed STD and global roles is dynamically guaranteed.

We have specified the Paradigm models of both variants of the email example from Section 2 in mCRL2 and verified a number of properties using the mCRL2

\footnotetext{
3 See www.mcrl2.org.
} 
toolset. Here, we list some of them. Note, the translation requires the double occurrence of label pmsg in the adapted STD for PDA to be distinguished. We use pmsg1 from Idle to Plain, and pmsg2 from Override to Plain.

(a) It is not possible to send a plain message while being outside the security perimeter. This property is expressed as

[ true*. sync(leave,triv). ( !sync(enter,triv) )* . sync(pmsg1,free) ] false

Here, sync(pmsg1,free) denotes an initiation of sending a message in plain mode, not triggered by overriding. sync(leave,triv) and sync(enter,triv) represent synchronization of the security module and the PDA in its role PDA(LoPo) captured by the consistency rules on page 7 . The above formula states that a sequence of actions in which sync(leave,triv) is followed by sync(pmsg1,free) is impossible if no action sync(enter,triv) is in between. The term (!sync(enter,triv))* expresses a sequence of actions different from sync(pmsg1,free). The tool reported this formula to be valid for both versions of the email example.

(b) For the second version of the email example, we have checked that a message in plain mode can be issued while the PDA is outside the security perimeter only if overriding has been requested. We use the synchronization action sync(pmsg2, free) to denote the request to override, and the local EMC action psend to denote the sending of a message in plain mode:

[ true*.sync1(leave,triv).

( !( sync(pmsg2,free) || psend || sync(enter,triv) ) )* . sync(pmsg1,free) .

( !( sync(pmsg2,free) \| psend \| sync(enter,triv) ) )* . psend ] false

A sequence of actions in which sync(leave,triv) is followed by psend, and in between sync(pmsg1,free) occurs, but neither sync(pmsg2, free), nor sync(enter,triv) nor psend occur, is impossible. In mCRL2, $\|$ denotes disjunction.

(c) If a sending of a message in a certain mode is initiated, assuming fairness, the sending event is executed eventually. For instance, an initiation of sending a message in plain mode, denoted by sync(pmsg1,free), will be followed by a sending event in plain mode, psend, i.e.

[ true* . sync(pmsg2,free) . ( !psend $\left.)^{*}\right]\langle$ psend $\rangle$ true

Similar formulas are checked for the other modes. Note, the 1-1-correspondence of initiation and actual sending, is guaranteed by the previous property.

We have translated the Paradigm model of the voting scheme of Section 3 into mCRL2 as well. ${ }^{4}$ For the base case of a single voter the generated LTS has about 130.000 states and 565.000 transitions. Specific tuning of the BES-solver was needed to cope with state space explosion in the case of multiple voters. However, the overall architecture, as represented in Paradigm, with its clear separation of phases and roles per component in each voting phase, allowed us to investigate certain properties of the protocol as a whole, by localizing them on the relevant components. Thus, we have been able to verify, by modularization, a number of security properties, as discussed below.

\footnotetext{
${ }^{4}$ See www.win.tue.nl/ andova/research/mcrl2-experiments/VotingExample/.
} 
(a) A voter without a signature from the administrator is not allowed to vote. And, if a voter has not been registered or has not voted, he cannot uncover. Partition AsVoter captures the behaviour of a voter and using the relevant trap information we express these properties as

[ true* . ( !ready_complete $)^{*}$. encDone ] false \&\& [ true* . ( !(ready_complete $\|$ encDone $))^{*}$. uncoverDone ] false

(b) If the voter for any reason is locked out, he cannot cast his vote. Using the synchronization between Human(AsVoter) and Administrator(AsServer), sync(ready, nosignature) and the voter action droppedOff, we can express this by the property

[ true* . sync(ready,nosignature) . (!encDone)* . encDone ] false \&\& [ true* . droppedOff . (!encDone)* . encDone ] false

(c) The next property reflects the coordination of the components of the scheme in the election phase, driven by the organizer. It states that: as soon as the first two phases are closed, i.e. no voter can be registered by the administrator, no voting is allowed anymore, neither he can cast his vote. As the closing of the voting phases is orchestrated by the organizer performing action proceed, we can express the property as

[ true* . sync(proceed, triv, triv, ready) . (!encDone)* . encDone] false

(d) The last property we consider is that no voter will be allowed to vote more than once. The property [ true*. sendEnc $i \cdot\left(\text { sendEnc }_{i}\right)^{*}$. sendEnc $i$ ] false is confirmed by the model checker. This means that a sequence of actions in which there are at least two occurrences of action sendEnc $i$ is not possible.

We briefly discuss malicious voter behavior in the FOO voting scheme. Any malicious activity a voter wants to perform, can be modeled as a local action in the detailed STD of Human, Figure 7. For instance, an additional outgoing transition from state WithForm or Filled back to state Invited means that the voter has an option to ask for a form more than once. Or, a transition from LockedOut back to Idle would mean that the voter may attempt to start the voting process again after he has been dismissed and put in state LockedOut. However, in the Paradigm model, the phases and traps chosen constrain the voter's global behaviour and prevent a dishonest voter to proceed from one to another voting phase as soon as he does not follow the voting policy and timely executes the steps required. For instance, take transition askFormAgain from state ToForm to state Invited. In the partition Human(AsVoter), we find that this transition may be possibly permitted in three phases. However, in the phases PreAdmin and LastPreAdmin the state ToForm forms a trap, thus missing any outgoing transitions. Furthermore, in phase Finished, the transition added does not play any role as the phase itself does not have any outgoing transitions. Thus, the transition askFormAgain does not add any behaviour to the voting scheme. Similarly, an additional transition from LockedOut to Invited at the detailed level does not add any global behaviour either.

Intuitively this means that as long as the voter executes these actions according to the voting policy, dishonest activities of the voter are irrelevant and are not a threat to the voting scheme. More precisely, by being in accordance to the 
voting policy, we actually mean to be branching bisimilar to the behaviour of the honest voter. In other words, if the relevant actions listed above are observed and all other local actions are hidden, both from the original model and from an extended or adapted model of a dishonest voter, then branching bisimilarity of the two detailed voter behaviours implies that both voters will show the same overall behaviour in the voting process. This, in fact, provides a security proof of the dishonest voter model with respect to the honest voter model, based on equivalence checking.

\section{Related work}

Since the seminal paper [31], tool-supported security protocol analysis has been flourishing. The tool Casper provides a high-level language for describing security protocols and secrecy or authentication properties together with a front-end for the CSP-based model checker FDR. For strand spaces, a framework of reconciling complete and partial protocol runs, the Athena tool [42] as well as the constraint solving approach of [36] are available for computer assistance. Another tool for the verification of security protocols is ProVerif [9]. More recent high-performance security checkers include the on-the-fly model checker [7] and the Scyther tool [12]. Main focus of these approaches is not so much the overall architecture, but rather secrecy and authentication in the small, the verification of secrecy and authentication properties of specific security protocols.

In the setting of formal description and analysis methods, anonymity of security protocols goes back to [38] dealing with the Chaum's famous Dining Cryptographers problem and proposing a notion of anonymity based on traceequivalence and invariance of permutation of agent names. The use of modal logics, to keep track dynamically of knowledge of principals underlies the approach of $[35,29,13]$, for example, for automated anonymity checking. Network anonymity, with the Crowds network as leading example, has been addressed in $[15,33,40]$. For the later case study, the Prism probabilistic model checker has been used. Anonymity for $\pi$-calculi has been proposed in [8], in combination with information hiding in [39]. In the present paper, following [33], anonymity is implied by a behavioral property, viz. strict separation of the stages of administering and encrypting from the stage of uncovering.

Access control policies can be integrated in UML models in the approach of [6], called Model Driven Security. The SecureUML proposed, supports various modeling techniques and transformation functions for the construction of access control structures. In [1], a framework is presented for programming distributed computer-supported cooperative work with regulation of role-based access control. In the RW framework [45] for generation and evaluation of access control policies a dedicated model checker can be used to assess policy compliance. Access control and security policies, as illustrated by the email example, can be modeled easily in Paradigm, but is not supported directly. Generic formalisms for architectural modeling, such as the higher-order architectural connectors of [30], can be instantiated to deal with security issues. 
Coordination languages can be divided into three main categories: databased, flow-based and transition-based. Some security issues, in particular rolebased access control and trust management, have been addressed in the context of data-based and transition-based coordination languages. Confidentiality in data-based, Linda-like coordination languages is mainly achieved via encryption of tuples and access control on the tuple space. In the context of agent systems, [37] proposes a framework for dynamically establishing security policies. Other approaches to tuple space security include SecOS [44] and SecSpace [11], which also come equipped with a process algebraic semantics. Role-based access control can be statically achieved in transition-based coordination settings via dedicated components. Dynamicity is much more subtle, cf. [10] for a $\pi$-calculus dialect. A calculus for ubiquitous computing dealing with trust is proposed in [26], a framework allowing LTL model checking. An instance of a formal approach based on the actor model dealing with trust management is [41]. Paradigm, also a transition-based coordination language, provides an architectural view on secure coordination, unlike the other example formalisms mentioned.

The separation of computation and coordination has been seen as a valuable concept. Flow-based coordination languages strictly follow this distinction: components comprise computation, streams and manipulations thereof through channels comprise coordination. Perhaps, such strong separation causes a gap difficult to bridge. For security problems at least, it is not so clear how honest or malicious dynamics within one component, via the flows the component brings about, must lead to or cannot lead to certain dynamics within another component. The problem then becomes, how to guarantee that component dynamics and the flows between them are coupled right and secure indeed.

\section{Concluding Remarks}

In brief, the approach outlined above constitutes a modeling suite for design and architecture of security solutions. The suite comprises Paradigm, process algebra and model checking. Paradigm provides the means to factorize security issues and other aspects into focused collaborations and protocols. In process algebra the reformulation of Paradigm models can be further molded using hiding and abstraction, relying on appropriate notions of process equivalence. The state-of-art mCRL2 toolset supports the analysis and formal verification of security properties and system requirements. The following observations particularly highlight the relevance of the modeling suite.

Separation of concerns helps greatly. As we have demonstrated, not only in the larger voting example, but also in the small email example, it worked out well to split the interaction into different protocols, yet remaining sufficiently consistent. This is complementary to the usual all-detail-matters attitude seen in security protocol analysis.

In the Paradigm visualizations above we did not try to follow UML 2.0 closely. We could have done much better in this respect, however, if not space restrictions had prevented us to do so. E.g. collaboration diagrams and activity diagrams could have been used. For instance, for each of the four protocols in Figures 4 
and 6 , we could have used a collaboration diagram. Also, for the consistency rules constituting one protocol, we could have used an activity diagram with swimlanes per role and per conductor. Although nicely clarifying, particularly the latter are not so small. See $[3,20]$ for such a stronger UML flavor. Additional sequence diagrams illustrating protocol interaction in greater detail, would have underlined similarities between our solution and the one in [32] visually too.

Translation of Paradigm models into process algebra [4] preserves the dynamic constraint compositions. Thus, phase and trap constraints as well as consistency rules remain embedded in the synchronization accordingly. By model checking with the mCRL2 tool set, we have succeeded in formally analyzing and verifying properties of an email security system as well as of the FOO e-voting scheme. In this manner, we have established validity of the original Paradigm models and their quality for security systems.

Future work includes the analysis of malicious behaviour. If in a setting the relevant interaction of a malicious agent is essentially the same as that of a honest one, i.e. if after hiding of internal actions their observed behaviour is branching bisimilar, the system is secure regarding dishonest principals. It is noted, such an approach can very well be combined with appropriate modularization guided by the Paradigm architecture, resulting in smaller state spaces to explore.

In a more general setting than security and architecture, other future work will be directed towards developing a tool suite supporting these ideas, amongst others. The tool suite aims at providing an integrated environment for editing and animating/running Paradigm models (cf. [43]), for reproducing and animating their dynamics in UML (cf. [20]), for translating them into PA and subsequently analyzing and model checking the resulting processes. As already touched upon in $[4,5]$, we particularly aim at feeding unwanted verification results back into the editing and animating parts of the tool suite.

Acknowledgments. We are grateful to Tim Willemse for advice on the use of the mCRL2 toolkit and to the ADS6-reviewers for their constructive comments.

\section{References}

1. T. Ahmed and A.R. Tripahti. Specification and verification of security requirements in a programming model for decentralized CSCW systems. Transactions on Information and Systems Security, 10, 2007. Article 7.

2. R. Anderson. A Guide to Building Dependable Distributed Systems. Wiley, 2001.

3. S. Andova, L. Groenewegen, and E. de Vink. System evolution by migration coordination. In A. Serebrenik, editor, Proc. BENEVOL 2008, pages 18-21, Eindhoven, 2008. Technische Universiteit Eindhoven.

4. S. Andova, L.P.J. Groenewegen, and E.P. de Vink. Dynamic consistency in process algebra: From Paradigm to ACP. In C. Canal, P. Poizat, and M. Sirjani, editors, Proc. FOCLASA'08. ENTCS, to appear. 19pp, extended version submitted.

5. S. Andova, L.P.J. Groenewegen, and E.P. de Vink. Formalizing adaptation onthe-fly. In G. Salaün and M. Sirjani, editors, Proc. FOCLASA'09. ENTCS, to appear.

6. D. Basin, J. Doser, and T. Lodderstedt. Model driven security: UML models to access control infrastructures. Transactions on Software Engineering and Methodology, 15:39-91, 2006. 
7. D.A. Basin, S. Mödersheim, and L. Viganò. OFMC: A symbolic model checker for security protocols. Journal of Information Security, 4:181-208, 2005.

8. M. Bhargava and C. Palamidessi. Probabilistic anonymity. In M. Abadi and L. de Alfaro, editors, Proc. CONCUR, pages 171-185. LNCS 3653, 2005.

9. B. Blanchet. An efficient cryptographic protocol verifier based on Prolog rules. In Proc. CSFW, Cape Breton, pages 82-96. IEEE, 2001.

10. C. Braghin, D. Gorla, and V. Sassone. Role-based access control for a distributed calculus. Journal of Computer Security, 14:113-155, 2006.

11. M. Bravetti, N. Busi, R. Gorrieri, R. Lucchi, and G. Zavattaro. Security issues in the tuple-space coordination model. In T. Dimitrakos and F. Martinelli, editors, Proc. FAST'04. Kluwer, 2005. 13pp.

12. C.J.F. Cremers. Scyther: Semantics and Verification of Security Protocols. PhD thesis, Technische Universiteit Eindhoven, 2006.

13. J. van Eijck and S. Orzan. Epistemic verification of anonymity. In M. ter Beek and F. Cadducci, editors, Proc. VODCA 2006, pages 159-174. ENTCS 168, 2007.

14. A. Fujioka, T. Okamoto, and K Ohta. A practical secret voting scheme for large scale elections. In J. Seberry and Y. Zheng, editors, Proc. ASIACRYPT'92, pages 244-251. LNCS 718, 1992.

15. F.D. Garcia, I. Hasuo, W. Pieters, and P. van Rossum. Provable anonymity. In V. Atluri, P. Samarati, R. Küsters, and J.C. Mitchell, editors, Proc. FMSE, Fairfax, pages 63-72. ACM, 2005.

16. D. Garlan. Software architecture: a roadmap. In Proc. ICSE 200, Limerick, pages 91-101. ACM, 2000.

17. L. Groenewegen and E. de Vink. Operational semantics for coordination in Paradigm. In F. Arbab and C. Talcott, editors, Proc. COORDINATION 2002, pages 191-206. LNCS 2315, 2002.

18. L. Groenewegen and E. de Vink. Evolution on-the-fly with Paradigm. In P. Ciancarini and H. Wiklicky, editors, Proc. COORDINATION 2006, pages 97-112. LNCS 4038, 2006.

19. L.P.J. Groenewegen, A.W. Stam, P.J. Toussaint, and E.P. de Vink. Paradigm as organization-oriented coordination language. In L. van de Torre and G. Boella, editors, Proc. CoOrg 2005, pages 93-113. ENTCS 150(3), 2005.

20. L.P.J. Groenewegen and E.P. de Vink. Dynamic system adaptation by constraint orchestration. Technical Report CSR 08/29, Department of Mathematics and Computer Science, Technische Universiteit Eindhoven, 2008. 20pp, arXiv:0811.3492v1.

21. J.F. Groote, A.H.J. Mathijssen, M.A. Reniers, Y.S. Usenko, and M.J. van Weerdenburg. The formal specification language mCRL2. In E. Brinksma, D. Harel, A. Mader, P. Stevens, and R. Wieringa, editors, Methods for Modelling Software Systems. IBFI, Schloss Dagstuhl, 2007. 34 pages.

22. J.F. Groote and M.A. Reniers. Algebraic process verification. In J.A. Bergstra, A. Ponse, and S.A. Smolka, editors, Handbook of Process Algebra, pages 1151-1208. Elsevier, 2001.

23. J.F. Groote and T. Willemse. Parameterised boolean equation systems. In Theoretical Computer Science, volume 343, pages 332-369, 2005.

24. J. Kramer, J. Magee, and S. Uchitel. Software architecture modeling \& analysis: A rigorous approach. In M. Bernardo and P. Inverardi, editors, SFM 2003, pages 44-51. LNCS 2804, 2003.

25. S. Kremer and M. Ryan. Analysis of an electronic voting protocol in the applied Pi calculus. In S. Sagiv, editor, Proc. ESOP, pages 186-200. LNCS 3444, 2005. 
26. K. Krukow, M. Nielsen, and V. Sassone. A logical framework for history-based access control and reputation systems. Journal of Computer Security, 16:63-101, 2008.

27. J. Küster. Consistency Management of Object-Oriented Behavioral Models. PhD thesis, University of Paderborn, 2004.

28. M. Lankhorst, editor. Enterprise Architecture at Work: Modelling, Communication and Analysis. Springer, 2005.

29. A. Lomuscio and F. Raimondi. MCMAS: A model checker for multi-agent systems. In H. Hermanns and J. Palsberg, editors, Proc. TACAS, pages 450-454. LNCS 3920, 2006.

30. A. Lopes, M. Wermelinger, and J.L Fiadeiro. Higher-order architectural connectors. Transactions on Software Engineering and Methodology, 12:64-104, 2003.

31. G. Lowe. Breaking and fixing the Needham-Schroeder public-key protocol using FDR. In T. Margaria and B. Steffen, editors, Proc. TACAS, pages 147-166. LNCS 1055, 1996.

32. S. Mauw, J. Verschuren, and E.P. de Vink. Data anonymity in the FOO voting scheme. In M. ter Beek and F. Gadducci, editors, Proc. VODCA 2006, pages 5-28. ENTCS 168, 2007.

33. S. Mauw, J. Verschuren, and E.P. de Vink. A formalization of anonymity and onion routing. In P. Samarati, P. Ryan, D. Gollmann, and R. Molva, editors, Proc. ESORICS 2004, pages 109-124, Sophia Antipolis, 2004. LNCS 3193.

34. S. Mauw, J. Verschuren, and E.P. de Vink. Data anonymity in the FOO voting scheme. In M. ter Beek and F. Gadducci, editors, Proc. VODCA 2006, pages 5-28. ENTCS 168, 2007.

35. R. van der Meyden and K. Su. Symbolic model checking the knowledge of the dining cryptographers. In Proc. CSFW, Pacific Grove, pages 280-291. IEEE, 2004.

36. J.K. Millen and V. Shmatikov. Constraint solving for bounded-process cryptographic protocol analysis. In Proc. CCS, Philadelphia, pages 166-175. ACM, 2001.

37. A. Omicini. Towards a notion of agent coordination context. In D.C. Marinescu and C. Lee, editors, Process Coordination and Ubiquitous Computing, chapter 12, pages 187-200. CRC Press, 2002.

38. S. Schneider and A. Sidiropoulos. CSP and anonymity. In E. Bertino, H. Kurth, G. Martella, and E. Montolivo, editors, Proc. ESORICS, pages 198218. LNCS 1146, 1996.

39. A. Serjantov and G. Danezis. Towards an information theoretic metric for anonymity. In R. Dingledine and P.F. Syverson, editors, Proc. PET, pages 4153. LNCS 2482, 2002.

40. V. Shmatikov. Probabilistic analysis of an anonymity system. Journal of Computer Security, 12:355-377, 2004.

41. V. Shmatikov and C.L. Talcott. Reputation-based trust management. Journal of Computer Security, 13:167-190, 2005.

42. D.X. Song, S. Berezin, and A. Perrig. Athena: A novel approach to efficient automatic security protocol analysis. Journal of Computer Security, 9:47-74, 2001.

43. A.W. Stam. Interaction Protocols in PARAdigm. PhD thesis, LIACS, Leiden University, 2009. Forthcoming.

44. J. Vitek, C. Bryce, and M. Oriol. Coordinating processes with secure spaces. Science of Computer Programming, 46:163-193, 2003.

45. N. Zhang, M. Ryan, and D.P. Guelev. Synthesising verified access control systems through model checking. Journal of Computer Security, 16:1-61, 2008. 